Article

\title{
Sequential Biotransformation of Antcin K by Bacillus subtilis ATCC 6633
}

\author{
Te-Sheng Chang ${ }^{1}{ }^{(1)}$, Chien-Min Chiang ${ }^{2}$, Yi-Yun Siao ${ }^{1}$ and Jiumn-Yih $\mathrm{Wu}^{3, *}$ \\ 1 Department of Biological Sciences and Technology, National University of Tainan, No. 33, Sec. 2, Shu-Lin St., \\ Tainan 70005, Taiwan; mozyme2001@gmail.com (T.-S.C.); nightzerow@gmail.com (Y.-Y.S.) \\ 2 Department of Biotechnology, Chia Nan University of Pharmacy and Science, No. 60, Sec. 1, Erh-Jen Rd., \\ Jen-Te District, Tainan 71710, Taiwan; cmchiang@mail.cnu.edu.tw \\ 3 Department of Food Science, National Quemoy University, No. 1, University Rd., Jin-Ning Township, \\ Kinmen County 892, Taiwan \\ * Correspondence: wujy@nqu.edu.tw; Tel.: +886-82-313310; Fax: +886-82-313797
}

Received: 6 August 2018; Accepted: 23 August 2018; Published: 27 August 2018

\begin{abstract}
The biotransformation of antcin $\mathrm{K}$, a major ergostane triterpenoid from the fruiting bodies of Antrodia cinnamomea, by Bacillus subtilis (B. subtilis) ATCC 6633 was studied. Four metabolites from the biotransformation were isolated with preparative high-performance liquid chromatography and identified as $25 S$-antcin $\mathrm{K} 26-\mathrm{O}-\beta$-glucoside, $25 \mathrm{R}$-antcin $\mathrm{K} 26-\mathrm{O}$ - $\beta$-glucoside, $25 S$-antcin $\mathrm{K}$ $26-O-\beta-\left(6^{\prime}-O\right.$-succinyl)-glucoside, and $25 R$-antcin $\mathrm{K} 26-O-\beta-\left(6^{\prime}-O\right.$-succinyl)-glucoside with mass and nuclear magnetic resonance spectral analysis. By using either $25 S$-antcin $\mathrm{K} 26-\mathrm{O}-\beta$-glucoside or $25 R$-antcin $\mathrm{K} 26-O-\beta$-glucoside as the biotransformation precursor, it was proven that $25 S$-antcin $\mathrm{K} 26-O-\beta$-(6'-O-succinyl)-glucoside and $25 R$-antcin $\mathrm{K} 26-O-\beta$ - $\left(6^{\prime}\right.$-O-succinyl)-glucoside were biotransformed from $25 S$-antcin $\mathrm{K} 26-\mathrm{O}-\beta$-glucoside and $25 \mathrm{R}$-antcin $\mathrm{K} 26-\mathrm{O}-\beta$-glucoside, respectively. To the best of our knowledge, this is the first study on the glycosylation of triterpenoids from $A$. cinnamomea, and the first time the succinylation of triterpenoid glycosides by microorganisms has been found. In addition, all four antcin $\mathrm{K}$ glucoside derivatives are new compounds.
\end{abstract}

Keywords: Antrodia cinnamomea; antcin K; Bacillus subtilis; biotransformation; glycosylation

\section{Introduction}

Antrodia cinnamomea is a well-known endemic Taiwanese medicinal mushroom, which has been used for the prevention or treatment of numerous diseases, including liver diseases, food and drug intoxication, diarrhea, abdominal pain, hypertension, itchy skin, and tumorigenic diseases [1]. Many of the constituents have been isolated from $A$. cinnamomea, including benzenoids, lignans, benzoquinones, polysaccharides, and terpenoids. Polysaccharides and terpenoids are considered the major bioactive compounds. A. cinnamomea contains two kinds of triterpenoids: lanostanes and ergostanes. Ergostanes mainly exist in the fruiting bodies of A. cinnamomea. Until now, dozens of ergostanes have been isolated and identified from the fruiting bodies of $A$. cinnamomea, and antcin $\mathrm{K}$ is one of the most abundant ergostane triterpenoids [2]. The compound was first isolated by Shen et al. [3] and has been proven to possess bioactivity, including anti-inflammatory, antidiabetic and antihyperlipidemic activities, the induction of apoptosis of hepatoma cells, and the reduction of carcinogenesis [4-8].

Searching for new compounds is very important for drug development. In addition to synthetic and natural sources, biotransformation offers an alternative strategy for obtaining new compounds. Xenobiotics can be biotransformed by cells, such as microorganisms, to form new compounds. Moreover, various modifications of the functional groups of xenobiotics during biotransformations often dramatically affect the bioactivity of the compounds. Due to the multiple bioactivities of 
triterpenoids, many scientists have studied the biotransformations of triterpenoids to obtain new bioactive triterpenoids from the biotransformation [9-11]. As described above, dozens of bioactive triterpenoids have been isolated from A. cinnamomea. However, there has been little research on the biotransformation of the triterpenoids from A. cinnamomea. Therefore, we are interested in the biotransformation of the triterpenoids from A. cinnamomea. Although polysaccharides and terpenoids have been identified in A. cinnamomea, interestingly, terpenoid glycosides have never been isolated from this species [1]. Accordingly, in the present study, we investigated the microbial-catalyzed glycosylation of triterpenoids from A. cinnamomea. The abundant ergostane triterpenoid antcin $\mathrm{K}$ from A. cinnamomea was used as a precursor, and one candidate microorganism Bacillus subtilis ATCC 6633 was selected to conduct the biotransformation of antcin $\mathrm{K}$. The biotransformation metabolites were then isolated and identified.

\section{Results and Discussion}

\subsection{Identifying Bacteria with Biotransformation Activity}

A great challenge for the realization of a desired biotransformation reaction is finding the appropriate microorganism. Three bacteria have been identified to catalyze glycosylation of triterpenoids [12-14]. Among them, B. subtilis ATCC 6633 [12] is available from the Bioresources Collection and Research Center (BCRC, Food Industry Research and Development Institute, Hsinchu, Taiwan). Thus, this strain was used to study the biotransformation of antcin $\mathrm{K}$ in the present study. The bacterium was then cultivated in broth with antcin $\mathrm{K}$, and the fermentation broth was analyzed using ultra-performance liquid chromatography (UPLC) to determine the ability of the strain to biotransform antcin $\mathrm{K}$.

Figure $1 \mathrm{a}, \mathrm{b}$ show the UPLC analysis of $0 \mathrm{~h}$ and $24 \mathrm{~h}$ fermentation broths of the strain $B$. subtilis ATCC 6633 fed with 25S-antcin K and 25R-antcin K, respectively. In Figure 1a, the precursor 25S-antcin $\mathrm{K}$ appears in the initial fermentation broth (dashed curves). After $24 \mathrm{~h}$ of fermentation, the peak of the precursor decreases, while two new peaks, compound (1) and compound (2) (solid curves), appear. In Figure 1b, 25R-antcin K appears in the initial fermentation broths (dashed curves). After $24 \mathrm{~h}$ of fermentation, the peak of the precursor decreases, while another two new peaks, compound (3) and compound (4) (solid curves), appear. Moreover, the four new peaks do not appear in the fermentation broths of the strain at $24 \mathrm{~h}$ in the absence of antcin K (Figure S1). The results imply that antcin K was digested by the strain to form biotransformation metabolites, and compound (1) and compound (2) were derived from 25S-antcin $\mathrm{K}$, while compound (3) and compound (4) were derived from $25 R$-antcin K.

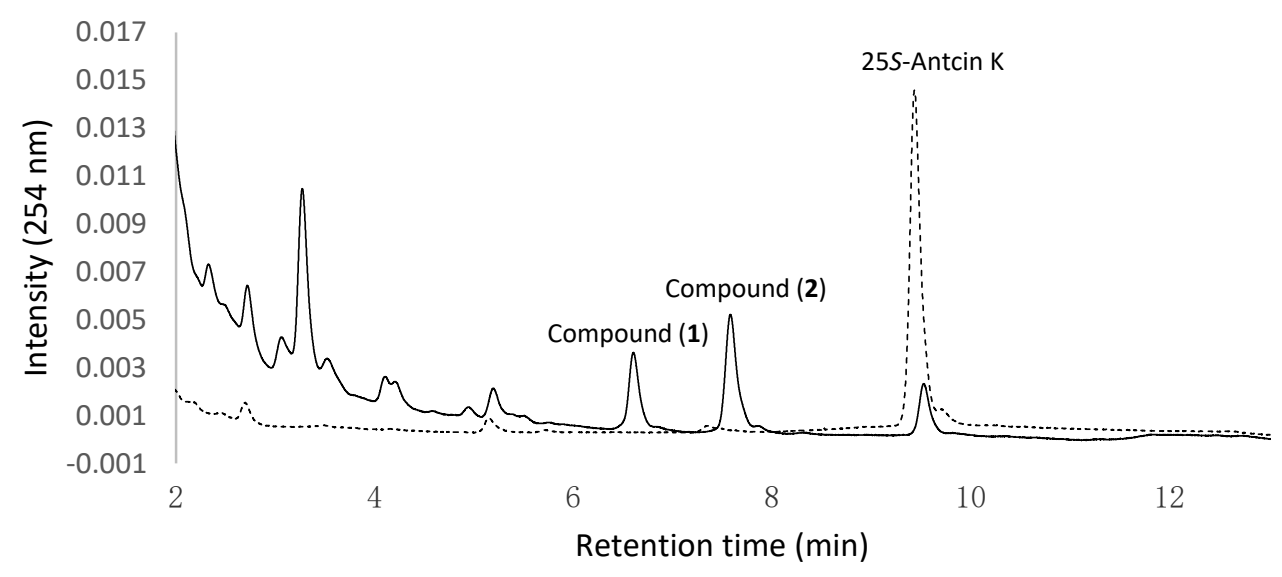

(a)

Figure 1. Cont. 


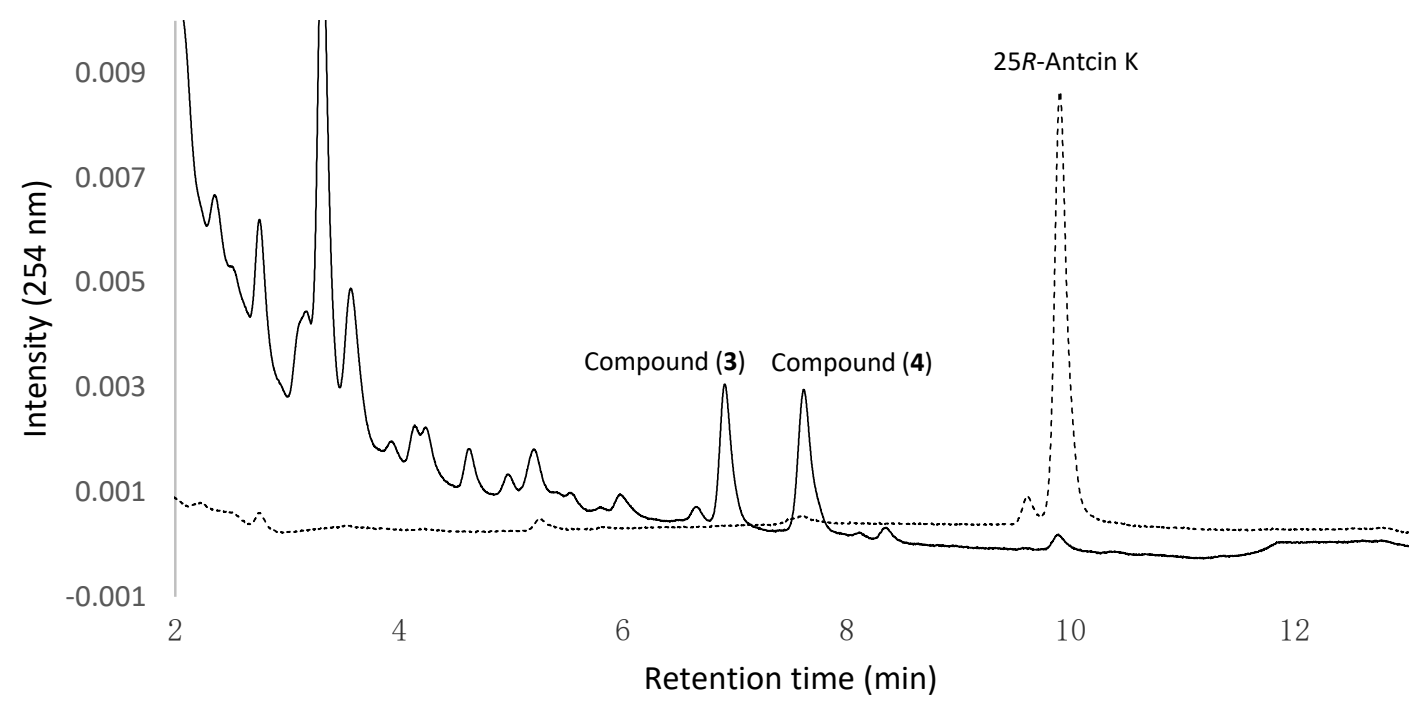

(b)

Figure 1. Biotransformation of antcin $\mathrm{K}$ by B. subtilis ATCC 6633. The strain was cultivated in modified glucose nutrient (MGN) media containing (a) $25 S$-antcin $\mathrm{K}$ or (b) $25 R$-antcin $\mathrm{K}$. The initial (dashed curves) and $24 \mathrm{~h}$ (solid curves) cultivations of the fermentation broths were analyzed with an UPLC system equipped with an Acquity ${ }^{\circledR}$ UPLC BEH C18 column (Acquity UPLC BEH C18, $1.7 \mu \mathrm{m}, 2.1$ i.d. $\times 100 \mathrm{~mm}$, Waters, Milford, MA, USA). The UPLC operation conditions are described in Materials and Methods.

\subsection{Isolation and Identification of Biotransformation Metabolites}

To resolve the chemical structures of the four metabolites, the biotransformation was scaled up, and the four metabolites were purified with preparative high-performance liquid chromatography (HPLC). From a 6.3-L fermentation broth containing $107 \mathrm{mg}$ antcin $\mathrm{K}, 9.6 \mathrm{mg}$ of compound (1), $11.3 \mathrm{mg}$ of compound (2), $6.1 \mathrm{mg}$ of compound (3), and $13.7 \mathrm{mg}$ of compound (4) were isolated. The chemical structures of the four compounds were identified using spectrophotometric methods. Compound (1) and compound (3) showed identical mass and nuclear magnetic resonance (NMR) spectrum data. They showed an $[\mathrm{M}+\mathrm{H}]^{+}$ion peak at $m / z: 651.66$ in the electrospray ionization mass (ESI-MS) spectrum corresponding to the molecular formula $\mathrm{C}_{35} \mathrm{H}_{54} \mathrm{O}_{11}$. Compound (2) and compound (4) showed identical mass and NMR spectrum data. They showed an $[\mathrm{M}-\mathrm{H}]^{-}$ion peak at $m / z: 749.56$ in the ESI-MS spectrum corresponding to the molecular formula $\mathrm{C}_{39} \mathrm{H}_{58} \mathrm{O}_{14}$.

A further detailed analysis of the ${ }^{1} \mathrm{H}$ - and ${ }^{13} \mathrm{C}-\mathrm{NMR}$ spectra and signal assignments, aided by distortionless enhancement by polarization transfer (DEPT), heteronuclear single quantum coherence (HSQC), heteronuclear multiple bond connectivity (HMBC), correlation spectroscopy (COSY), and nuclear Overhauser effect spectroscopy (NOESY) experiments, was performed to elucidate the structure of compounds (1) to (4) (shown in Figures S2-S13 and Table S1). The ${ }^{13} \mathrm{C}-\mathrm{NMR}$ spectrum of compound (1) showed, in addition to 29 signals consistent with the triterpenoid structure of antcin $\mathrm{K}[3]$, six peaks in the range at $\delta 62-97 \mathrm{ppm}(96.3,74.2,78.7,71.1,79.5$, and $62.3 \mathrm{ppm})$ corresponding to the presence of a glucose moiety [15]. The glucose proton signals were assigned using the $\mathrm{H}, \mathrm{H}-\mathrm{COSY}$ experiment. The common D-configuration for glucose was assumed according to what was most commonly encountered among the glycosides transformed by this microorganism. The anomeric proton signal at $\delta 6.40(\mathrm{~J}=8.2 \mathrm{~Hz})$ in the ${ }^{1} \mathrm{H}$-NMR spectrum indicated the $\beta$-configuration for the glucopyranosyl moiety. In the HMBC experiment, the anomeric proton peak at $\delta 6.40$ correlated with the carboxyl group of antcin $\mathrm{K}$ at $\delta 173.7$, which is the ${ }^{13} \mathrm{C}-\mathrm{NMR}$ signal of $\mathrm{C}-26$. Thus, the glycosidic linkage site of $\beta$-D-glucose was determined to be C-26. Therefore, compound (1)/(3) was determined to be $25 S / R$-antcin K $26-O-\beta$-glucoside. 
The ${ }^{1} \mathrm{H}$ - and ${ }^{13} \mathrm{C}$-NMR spectral data of compound (2)/(4) showed compound (1)/(3) with an extra succinyl group ( ${ }^{13} \mathrm{C}-\mathrm{NMR}: 174.5,172.8,29.5$, and $\left.29.5 \mathrm{ppm}\right)$. The downfield shift of glucose 6-protons (4.88 and $4.80 \mathrm{ppm}$ ) and 6-carbon (64.2 ppm), as well as the cross-peaks at 4.80/172.8 and 4.80/172.8 ppm in the HMBC spectrum, demonstrated the linkage of the succinyl group to the glucose 6 position in compound (2). Based on the evidence above, compound (2)/(4) was characterized accordingly as $25 S / R$-antcin $\mathrm{K} 26-O-\beta-\left(6^{\prime}-O\right.$-succinyl)-glucoside. This is the first report of their occurrence.

The structure and the key HMBC correlations of compounds $(\mathbf{1}) /(3)$ and $(2) /(4)$ are shown in Figure 2, and the spectroscopic data are listed in Table S1.

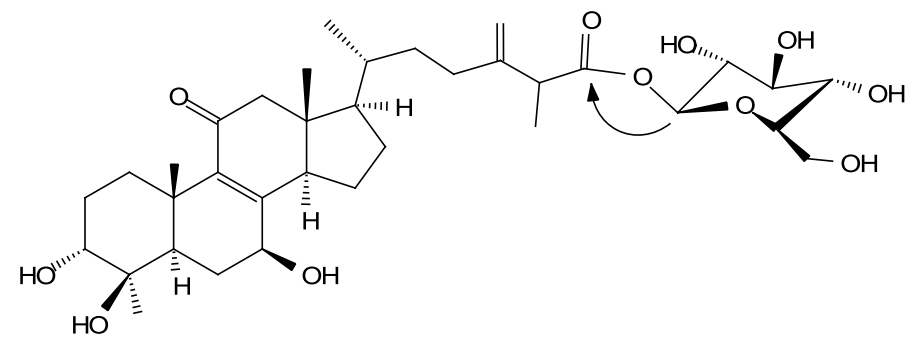

(a)

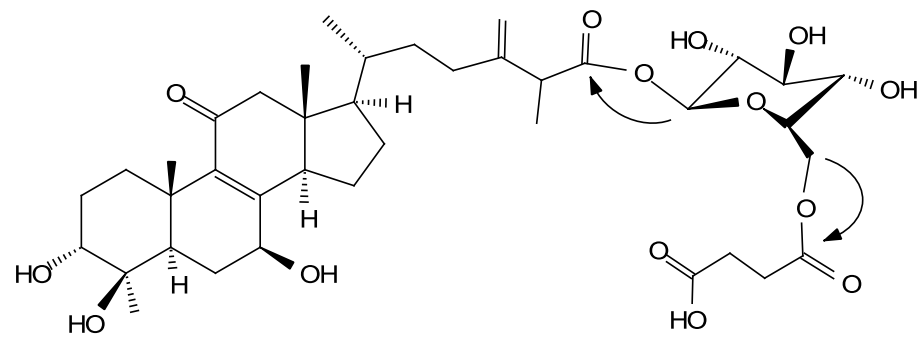

(b)

Figure 2. The structure and the key HMBC correlations (arrows) of compound (1)/(3) (a) and compound $(2) /(4)(b)$.

Unlike many of the triterpenoid glycosides isolated from plants, such as ginsenosides from ginseng, previously no triterpenoid glycoside had been isolated from A. cinnamomea. A possible reason for this is that plants are autotrophs and synthesize glucose from photosynthesis, while fungi are heterotrophs and obtain glucose from their environments. Glucose is usually not limited in plants, however it is in fungi. Therefore, the endogenous biosynthesis pathways of triterpenoids in plants favor the involvement of glycosylation, but not in fungi. Accordingly, developing a biotransformation system of microbial-catalyzed glycosylation, which could expand the molecule diversity and functionality of the unique bioactive triterpenoids from A. cinnamomea, would be interesting. Until now, all three of the bacteria strains identified to catalyze the glycosylation of triterpenoids favor catalyzing glucosylation at the C-28 carboxyl group of pentacyclic triterpenoids [12-14]. The structure of the precursor, antcin K, used in the present study, contains three hydroxyl groups, C-3, C-4, and C-7, and a C-26 carboxyl group. In the results of the present study, only the $\mathrm{C}-26$ carboxyl group of antcin $\mathrm{K}$ was glucosylated during the biotransformation. The results revealed that B. subtilis ATCC 6633 favors catalyzing glucosylation at a carboxyl group site, which is consistent with those of previous studies [12-14].

\subsection{Identification of the Biotransformation Process}

From the structures of the four metabolites, where compound (2) and compound (4) are succinyl derivatives of compound (1) and compound (3), respectively, it seems that compound (2) and compound (4) were biotransformation metabolites from compound (1) and compound (3), respectively. To resolve the biotransformation process, either compound (1) or compound (3) was used as the 
precursor to conduct the biotransformation experiments. Figure 3 shows the UPLC analysis of initial (dash curves) and $24 \mathrm{~h}$ (solid curves) fermentation broths of B. subtilis ATCC 6633 in the presence of compound (1) (Figure 3a) or compound (3) (Figure 3b). Purified compound (2) (Figure 3c) and compound (4) (Figure 3d) were used as standards. In the results, compound (2) and compound (4) appeared in the $24 \mathrm{~h}$ fermentation broth with the feeding of compound (1) and compound (3), respectively. Thus, it was concluded that compound (2) and compound (4) were biotransformation metabolites from compound (1) and compound (3), respectively. Figure 4 shows the biotransformation process of antcin K by the B. subtilis ATCC 6633 strain.

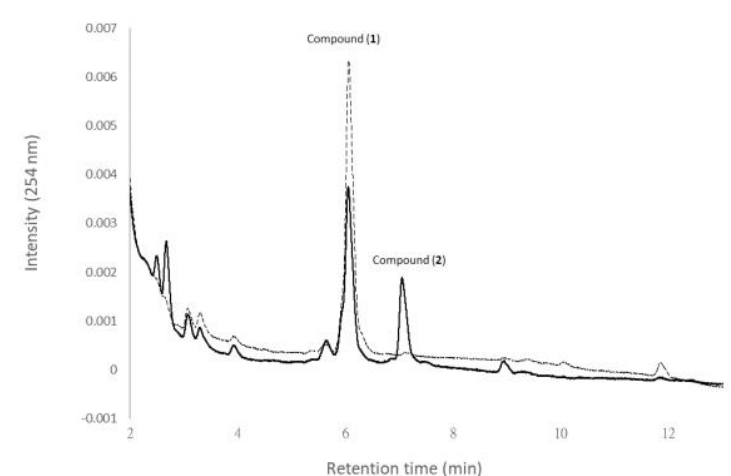

(a)

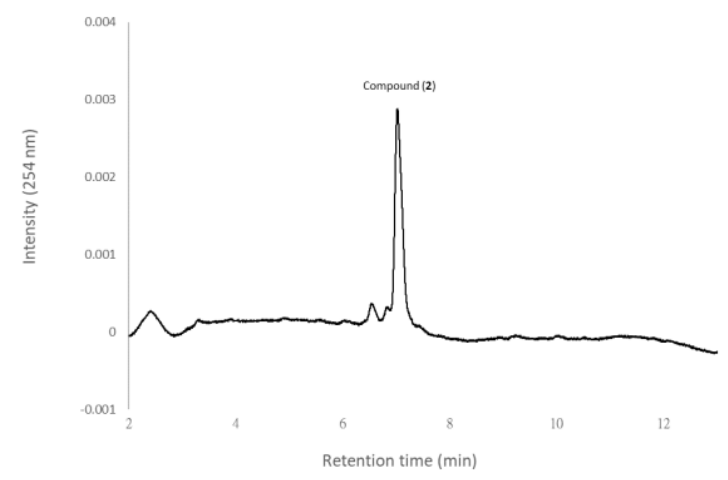

(c)

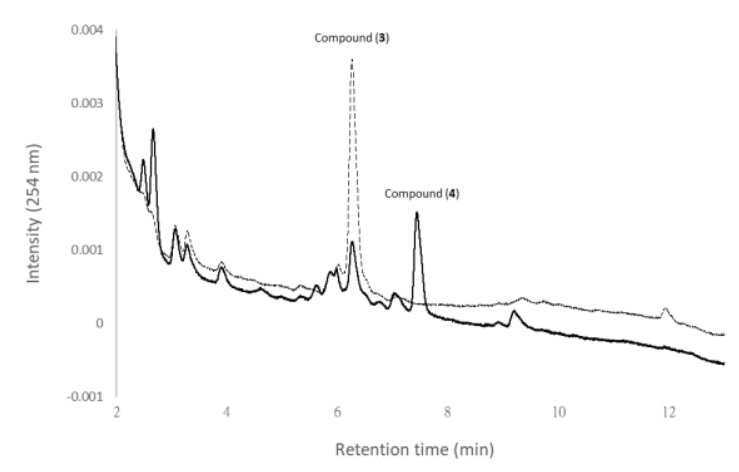

(b)

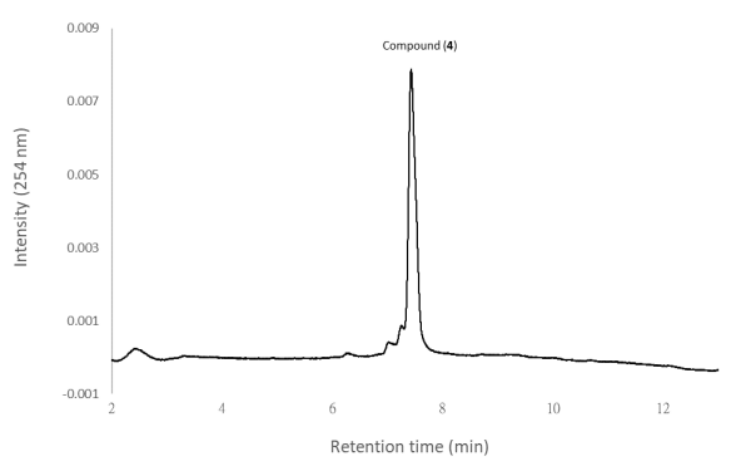

(d)

Figure 3. Biotransformation of compound (1) and compound (3) by B. subtilis ATCC 6633. The strain was cultivated in MGN media containing compound (1) (a) and compound (3) (b). The initial (dash curves) and $24 \mathrm{~h}$ (solid curves) cultivations of the fermentation broths were analyzed with a UPLC system equipped with a Kinetex ${ }^{\circledR}$ UPLC C18 column $(1.7 \mu \mathrm{m}, 2.1$ i.d. $\times 100 \mathrm{~mm}$, Phenomenex Inc., Torrance, CA, USA). Purified compound (2) (c) and compound (4) (d) were used as standards. The UPLC operation conditions are described in Materials and Methods.

Toda et al. [16] and Park [17] reported that the Bacillus genus had succinylation activity toward flavonoid glucosides. Toda et al. first demonstrated that soy isoflavone glucosides, inducing daidzin and genistin, were biotransformed into 6"-succinyl glucoside forms by B. subtilis (natto) during soybean fermentation [16]. Park et al. proved that B. subtilis (natto) and B. subtilis catalyze 6 "-succinylation of soy isoflavone glucosides, daidzin and genistin, while the two strains could not catalyze 6"-succinylation of soy isoflavone aglycon, daidzein and genistein [17]. It is reasonable that 6-succinyl glucosides are not produced from the direct glycosylation of aglycon formed by B. subtilis. In nature, enzyme-catalyzed glycosylation is conducted by glycosyltransferases (GTs, EC 2.4.x.y), which transfer sugar moieties from the activated donor molecules to specific acceptor molecules [18-20]. More than 10,000 GTs have been identified. However, no GT has been proven to directly transfer 6-succinyl glucosides to acceptor molecules. Therefore, 6-succinyl glucosides should 
be produced from the succinylation of the corresponding glucosides. The present results demonstrating that $25 S$-antcin $\mathrm{K} 26-\mathrm{O}-\beta-\left(6^{\prime}-\mathrm{O}\right.$-succinyl)-glucoside and $25 \mathrm{R}$-antcin $\mathrm{K} 26-\mathrm{O}-\beta-\left(6^{\prime}-\mathrm{O}\right.$-succinyl)-glucoside were biotransformed from $25 S$-antcin K $26-O-\beta$-glucoside and $25 R$-antcin K $26-O-\beta$-glucoside, respectively, were also consistent with the findings above. In addition, from the present results and previous ones, B. subtilis seems to contain the enzymes necessary for catalyzing 6-succinylation of the corresponding glucosides. However, it is unclear whether the enzymes for catalyzing soy isoflavone glucosides and triterpenoid glucosides are the same. Moreover, B. subtilis ATCC 6633 has been proven to biotransform glucosylation of the pentacyclic triterpenoids oleanolic acid, echinocystic acid, and betulinic acid [12]. However, no $6^{\prime}$-succinyl glucoside derivatives were found in that study. Whether the enzyme catalyzing $6^{\prime}$-succinylation of antcin K glucosides can catalyze those triterpenoid glucosides is an interesting issue. Further study is needed to investigate the mechanism involved.

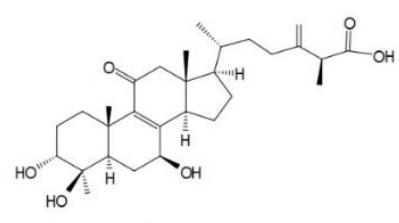

25S-Antcin K

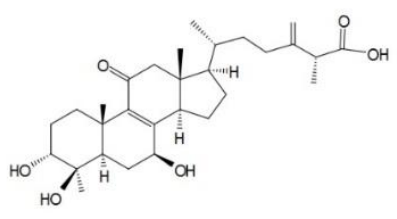

25R-Antcin K

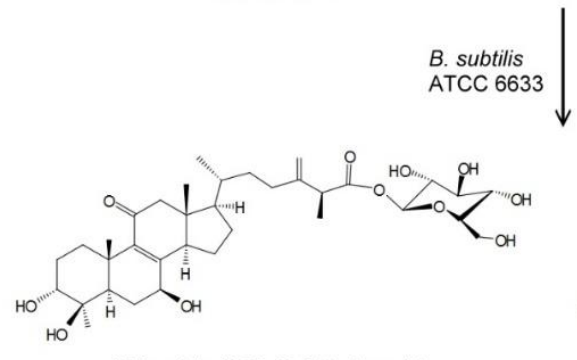

25S-antcin K 26-O- $\beta$-D-glucoside

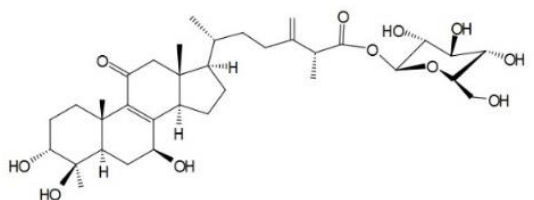

$25 R$-antcin K 26-O- $\beta$-D-glucoside

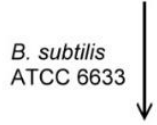

6'-O-succinylation of antcin $\mathrm{K}$ glucosides

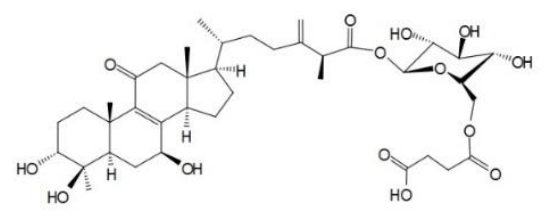

25S-antcin K 26-O- $\beta$-(6'-O-succinyl)-D-glucoside

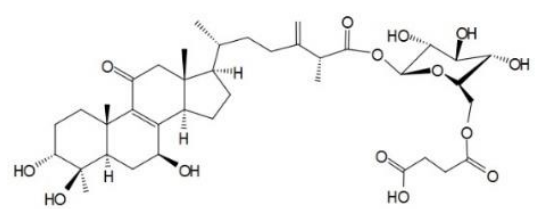

$25 R$-antcin K 26-O- $\beta$-(6'-O-succinyl)-D-glucoside

Figure 4. Biotransformation process of antcin K by the B. subtilis ATCC 6633 strain.

\section{Materials and Methods}

\subsection{Microorganism and Chemicals}

B. subtilis ATCC 6633 (BCRC 10447) [12] was purchased from BCRC (Food Industry Research and Development Institute, Hsinchu, Taiwan). All chemical reagents and solvents used were of high quality and were purchased from commercially available sources.

\subsection{Preparation of Antcin $K$}

Antcin $\mathrm{K}$ was prepared according to the procedures in our previous study [21]. Briefly, an ergostane-enriched fraction extracted from $100 \mathrm{~g}$ of dried dish-cultivated A. cinnamomea (Honest \& Humble Biotechnology Co., Ltd., New Taipei City, Taiwan) by 50\% methanol was subjected to preparative HPLC to obtain $1.2 \mathrm{~g}$ of antcin $\mathrm{K}$. Then 13.5 and $23.7 \mathrm{mg}$ of $25 \mathrm{~S}$ - and $25 R$-epimers of antcin $\mathrm{K}$ were further purified from $70 \mathrm{mg}$ of antcin $\mathrm{K}$ with preparative HPLC using the reverse-phase C-18 column (Inertsil ODS-3, $10 \mu \mathrm{m}, 20.0$ i.d. $\times 250 \mathrm{~mm}$ ) from GL Sciences Inc. (Tokyo, Japan). 


\subsection{Identification Bacteria with Biotransformation Activity}

B. subtilis ATCC 6633 was cultivated in a $250-\mathrm{mL}$ baffled Erlenmeyer flask containing $20 \mathrm{~mL}$ of a modified glucose-nutrient (MGN) medium ( $5 \mathrm{~g} / \mathrm{L}$ of peptone, yeast extract, $\mathrm{K}_{2} \mathrm{HPO}_{4}$, and $\mathrm{NaCl}$; $20 \mathrm{~g} / \mathrm{L}$ of glucose) [13] and $17 \mathrm{mg} / \mathrm{L}$ of antcin $\mathrm{K}, 25 \mathrm{~S}$-antcin $\mathrm{K}$, or 25- $R$ antcin K. After cultivation at $180 \mathrm{rpm}, 28^{\circ} \mathrm{C}$ for $12 \mathrm{~h}$ or $24 \mathrm{~h}, 1 \mathrm{~mL}$ of the culture was then mixed with an equal volume of methanol. The cell debris was removed by centrifugation at $10,000 \times g$ for $10 \mathrm{~min}$. The supernatant from the extracted broth was assayed with UPLC to measure the biotransformation activity.

\subsection{UPLC Analysis}

The UPLC system (Acquity UPLC H-Class, Waters, Milford, MA, USA) was equipped with an analytic C18 reversed-phase column from Waters (Acquity UPLC BEH C18, $1.7 \mu \mathrm{m}, 2.1$ i.d. $\times 100 \mathrm{~mm}$ ) or from Phenomenex Inc. (Kinetex ${ }^{\circledR}$ C18, $1.7 \mu \mathrm{m}, 2.1$ i.d. $\times 100$ mm, Phenomenex Inc., Torrance, CA, USA). The operation conditions for UPLC analysis were modified from those in our previous study [21], where a gradient elution using water (A) containing $1 \%(v / v)$ acetic acid and methanol (B) with a linear gradient for 11 min with $50 \%$ to $80 \%$ B and for another 2 min with $80 \%$ to $100 \%$ B was conducted at a flow rate of $0.2 \mathrm{~mL} / \mathrm{min}$, an injection volume of $0.2 \mu \mathrm{L}$, and absorbance detection at $254 \mathrm{~nm}$.

\subsection{Scale-Up Fermentation, Isolation, and Identification of the Biotransformation Products}

The ATCC 6633 strain was cultured in a $1000 \mathrm{~mL}$ baffled Erlenmeyer flask containing $150 \mathrm{~mL}$ of MGN medium and $17 \mathrm{mg} / \mathrm{L}$ of antcin K at $180 \mathrm{rpm}, 28^{\circ} \mathrm{C}$ for $24 \mathrm{~h}$. A total of 42 flasks of ATCC 6633 cultivation $(6.3 \mathrm{~L})$ were conducted. After cultivation, the $6.3 \mathrm{~L}$ of fermentation broth were combined and extracted with $1 \mathrm{~L}$ of butanol twice. The butanol fractions were combined and condensed under a vacuum. The residue was then suspended in $200 \mathrm{~mL}$ of $50 \%$ methanol. After filtration through a $0.2 \mu \mathrm{m}$ nylon membrane, the suspension was injected into a preparative YoungLin HPLC system (YL9100, YL Instrument, Gyeonggi-do, South Korea). The system was equipped with a preparative C18 reverse-phase column (Inertsil ODS-3, $10 \mu \mathrm{m}, 20.0$ i.d. $\times 250 \mathrm{~mm}$ ). The operational conditions for the preparative HPLC analysis were the same as those in the UPLC analysis. The elution corresponding to the four peaks of the metabolites in the UPLC analysis were collected, concentrated under vacuum, and then lyophilized. Finally, $9.6 \mathrm{mg}$ of compound (1), $11.3 \mathrm{mg}$ of compound (2), $6.9 \mathrm{mg}$ of compound (3), and $13.7 \mathrm{mg}$ of compound (4) were obtained, and the structures of the compounds were confirmed with NMR and mass spectral analysis. The mass analysis was performed on a Finnigan LCQ Duo mass spectrometer (ThermoQuest Corp., San Jose, CA, USA) with electrospray ionization (ESI). ${ }^{1} \mathrm{H}$ - and ${ }^{13}$ C-NMR, DEPT, HSQC, HMBC, COSY, and NOESY spectra were recorded on a Bruker AV-700 NMR spectrometer (Bruker Corp., Billerica, MA, USA) at ambient temperature. Standard pulse sequences and parameters were used for the NMR experiments, and all chemical shifts were reported in parts per million $(\mathrm{ppm}, \delta)$.

\section{Conclusions}

The present study demonstrated the sequential biotransformation of $25 S$-antcin $\mathrm{K}$ and $25 R$-antcin $\mathrm{K}$ to $25 S$-antcin $\mathrm{K} 26-\mathrm{O}-\beta$-glucoside and $25 \mathrm{R}$-antcin $\mathrm{K} 26-\mathrm{O}-\beta$-glucoside at first, and then to $25 \mathrm{~S}$-antcin $\mathrm{K} 26-O-\beta$ - $\left(6^{\prime}-O\right.$-succinyl)-glucoside and $25 R$-antcin $\mathrm{K} 26-O-\beta-\left(6^{\prime}-O\right.$-succinyl)-glucoside by $B$. subtilis ATCC 6633 . To the best of our knowledge, this is the first study on glycosylation of triterpenoids from A. cinnamomea, and the first time that the succinylation of triterpenoid glycosides by microorganisms was found. In addition, all four antcin K glucoside derivatives are new compounds. 
Supplementary Materials: The following are available online at http://www.mdpi.com/2073-4344/8/9/349/s1, Table S1: NMR spectroscopic data for compound (1) and compound (2) (in pyridine- $d_{5} ; 700 \mathrm{MHz}$ ). Figure S1: The UPLC analysis of $24 \mathrm{~h}$ fermentation broth of $B$. subtilis ATCC 6633 in the absence of antcin K. Figure S2: The ${ }^{1} \mathrm{H}-\mathrm{NMR}\left(700 \mathrm{MHz}\right.$, Pyridine- $\left.d_{5}\right)$ spectrum of compound (1). Figure S3: The ${ }^{13} \mathrm{C}-\mathrm{NMR}\left(176 \mathrm{MHz}\right.$, Pyridine- $\left.d_{5}\right)$ spectrum of compound (1). Figure S4: The DEPT-90 and DEPT-135 (176 MHz, Pyridine- $\left.d_{5}\right)$ spectra of compound (1). Figure S5: The HSQC (700 MHz, Pyridine- $\left.d_{5}\right)$ spectrum of compound (1). Figure S6: The HMBC (700 MHz, Pyridine- $\left.d_{5}\right)$ spectrum of compound (1). Figure S7: The H-H COSY (700 MHz, Pyridine- $\left.d_{5}\right)$ spectrum of compound (1). Figure S8: The ${ }^{1} \mathrm{H}-\mathrm{NMR}$ (700 MHz, Pyridine- $\left.d_{5}\right)$ spectrum of compound (2). Figure S9: The ${ }^{13} \mathrm{C}-\mathrm{NMR}$ (176 MHz, Pyridine- $d_{5}$ ) spectrum of compound (2). Figure S10: The DEPT-90 and DEPT-135 (176 MHz, Pyridine- $\left.d_{5}\right)$ spectra of compound (2). Figure S11: The HSQC (700 MHz, Pyridine- $d_{5}$ ) spectrum of compound (2). Figure S12: The HMBC (700 MHz, Pyridine- $\left.d_{5}\right)$ spectrum of compound (2). Figure S13: The H-H COSY (700 MHz, Pyridine- $\left.d_{5}\right)$ spectrum of compound (2).

Author Contributions: Conceptualization, T.-S.C.; Data curation, T.-S.C., C.-M.C., Y.-Y.S. and J.-Y.W.; Methodology, T.-S.C., C.-M.C., Y.-Y.S. and J.-Y.W.; Project administration, T.-S.C.; Writing-original draft, T.-S.C., C.-M.C. and J.-Y.W.; Writing-review \& editing, T.-S.C., C.-M.C. and J.-Y.W.

Funding: This research was financially supported by grants from the National Scientific Council of Taiwan (Project No. MOST 107-2221-E-024-005-).

Conflicts of Interest: The authors declare no conflicts of interest.

\section{References}

1. Geethangili, M.; Tzeng, Y.-M. Review of pharmacological effects of antrodia camphorata and its bioactive compounds. Evid.-Based Complement. Altern. Med. 2011, 2011, 1-17. [CrossRef] [PubMed]

2. Lu, M.-C.; El-Shazly, M.; Wu, T.-Y.; Du, Y.-C.; Chang, T.-T.; Chen, C.-F.; Hsu, Y.-M.; Lai, K.-H.; Chiu, C.-P.; Chang, F.-R.; et al. Recent research and development of Antrodia cinnamomea. Pharmacol. Ther. 2013, 139, 124-156. [CrossRef] [PubMed]

3. Shen, C.C.; Kuo, Y.C.; Huang, R.L.; Lin, L.C.; Don, M.J.; Chang, T.T.; Chou, C.J. New ergostane and lanostane from Antrodia camphorata. J. Chin. Med. 2003, 14, 247-258. [CrossRef] [PubMed]

4. Geethangili, M.; Fang, S.-H.; Lai, C.-H.; Rao, Y.K.; Lien, H.-M.; Tzeng, Y.-M. Inhibitory effect of Antrodia camphorata constituents on the helicobacter pylori-associated gastric inflammation. Food Chem. 2010, 119, 149-153. [CrossRef]

5. Lai, C.-I.; Chu, Y.-L.; Ho, C.-T.; Su, Y.-C.; Kuo, Y.-H.; Sheen, L.-Y. Antcin K, an active triterpenoid from the fruiting bodies of basswood cultivated Antrodia cinnamomea, induces mitochondria and endoplasmic reticulum stress-mediated apoptosis in human hepatoma cells. J. Tradit. Complement. Med. 2016, 6, 48-56. [CrossRef] [PubMed]

6. Huang, Y.-L.; Chu, Y.-L.; Ho, C.-T.; Chung, J.-G.; Lai, C.-I.; Su, Y.-C.; Kuo, Y.-H.; Sheen, L.-Y. Antcin $\mathrm{K}$, an active triterpenoid from the fruiting bodies of basswood-cultivated Antrodia cinnamomea, inhibits metastasis via suppression of integrin-mediated adhesion, migration, and invasion in human hepatoma cells. J. Agric. Food Chem. 2015, 63, 4561-4569. [CrossRef] [PubMed]

7. Tien, A.J.; Chien, C.Y.; Chen, Y.H.; Lin, L.C.; Chien, C.T. Fruiting bodies of Antrodia cinnamomea and its active triterpenoid, antcin $\mathrm{K}$, ameliorates $N$-nitrosodiethylamine-induced hepatic inflammation, fibrosis and carcinogenesis in rats. Am. J. Chin. Med. 2017, 45, 173-198. [CrossRef] [PubMed]

8. Kuo, Y.H.; Lin, C.H.; Shih, C.C.; Yang, C.S. Antcin K, a triterpenoid compound from Antrodia camphorate, displays antidiabetic and antihyperlipidemic effects via glucose transporter 4 and AMP-activated protein kinase phosphorylation in muscles. Evid.-Based Complement. Altern. Med. 2016. [CrossRef] [PubMed]

9. Sultana, N.; Saify, Z.S. Enzymatic biotransformation of terpenes as bioactive agents. J. Enzym. Inhib. Med. Chem. 2012, 28, 1113-1128. [CrossRef] [PubMed]

10. Muffler, K.; Leipold, D.; Scheller, M.-C.; Haas, C.; Steingroewer, J.; Bley, T.; Neuhaus, H.E.; Mirata, M.A.; Schrader, J.; Ulber, R. Biotransformation of triterpenes. Process Biochem. 2011, 46, 1-15. [CrossRef]

11. Parra, A.; Rivas, F.; Garcia-Granados, A.; Martinez, A. Microbial transformation of triterpenoids. Mini-Rev. Org. Chem. 2009, 6, 307-320. [CrossRef]

12. Wang, W.W.; Xu, S.H.; Zhao, Y.Z.; Zhang, C.; Zhang, Y.Y.; Yu, B.Y.; Zhang, J. Microbial hydroxylation and glycosylation of pentacyclic triterpenes as inhibitors on tissue factor procoagulant activity. Bioorg. Med. Chem. Lett. 2017, 27, 1026-1030. [CrossRef] [PubMed] 
13. Zhang, C.; Xu, S.H.; Ma, B.L.; Wang, W.W.; Yu, B.Y.; Zhang, J. New derivatives of ursolic acid through the biotransformation by Bacillus megaterium CGMCC 1.1741 as inhibitors on nitric oxide production. Bioorg. Med. Chem. Lett. 2017, 27, 2575-2578. [CrossRef] [PubMed]

14. Feng, X.; Zou, Z.; Fu, S.; Sun, L.; Su, Z.; Sun, D.-A. Microbial oxidation and glucosidation of echinocystic acid by Nocardia corallina. J. Mol. Catal. B: Enzym. 2010, 66, 219-223. [CrossRef]

15. Agrawal, P.K. NMR spectroscopy in the structural elucidation of oligosaccharides and glycosides. Phytochemistry 1992, 31, 3307-3330. [CrossRef]

16. Toda, T.; Uesugi, T.; Hirai, K.; Nukaya, H.; Tsuji, K.; Ishida, H. New 6-O-Acyl Isoflavone Glycosides from Soybeans Fermented with Bacillus subtilis (natto). I. 6-O-Succinylated Isoflavone Glycosides and Their Preventive Effects on Bone Loss in Ovariectomized Rats Fed a Calcium-Deficient Diet. Biol. Pharm. Bull. 1999, 22, 1193-1201. [CrossRef] [PubMed]

17. Park, C.U.; Jeong, M.K.; Park, M.H.; Yeu, J.D.; Park, M.S.; Kim, M.S.; Kim, M.J.; Ahn, S.M.; Chang, P.S.; Lee, J.H. Formation of succinyl genistin and succinyl daidzin by Bacillus species. J. Food Sci. 2010, 75, C128-C133. [CrossRef] [PubMed]

18. Tiwari, P.; Sangwan, R.S.; Sangwan, N.S. Plant secondary metabolism linked glycosyltransferases: An update on expanding knowledge and scopes. Biotechnol. Adv. 2016, 34, 716-739. [CrossRef] [PubMed]

19. Kim, B.G.; Yang, S.M.; Kim, S.Y.; Cha, M.N.; Ahn, J.-H. Biosynthesis and production of glycosylated flavonoids in Escherichia coli: Current state and perspectives. Appl. Microbiol. Biotechnol. 2015, 99, 2979-2988. [CrossRef] [PubMed]

20. Hofer, B. Recent developments in the enzymatic O-glycosylation of flavonoids. Appl. Microbiol. Biotechnol. 2016, 100, 4269-4281. [CrossRef] [PubMed]

21. Chiang, C.M.; Wang, T.Y.; Ke, A.N.; Chang, T.S.; Wu, J.Y. Biotransformation of ergostane triterpenoid antcin K form Antrodia cinnamomea by soil-isolated Psychrobacillus sp. AK 1817. Catalysts 2017, 7, 299. [CrossRef]

(C) 2018 by the authors. Licensee MDPI, Basel, Switzerland. This article is an open access article distributed under the terms and conditions of the Creative Commons Attribution (CC BY) license (http://creativecommons.org/licenses/by/4.0/). 